in liver and intestine implicate abnormal hepcidin and Cybrd 1 expression in mouse hemochromatosis. Nat. Genet. 34:102-107.

13. Adamsky, K., et al. 2004. Decreased hepcidin mRNA expression in thalassemic mice. Br.J. Haematol. 124:123-124.

14. Dallalio, G., Fleury, T., and Means, R.T. 2003.
Serum hepcidin in clinical specimens. Br. J. Haematol. 122:996-1000.

15. Roetto, A., et al. 2003. Mutant antimicrobial peptide hepcidin is associated with severe juvenile hemochromatosis. Nat. Genet. 33:21-22.

16. Nicolas, G., et al. 2001. Lack of hepcidin gene expression and severe tissue iron overload in upstream stimulatory factor 2 (USF2) knockout mice. Proc. Natl. Acad. Sci. U. S. A. 98:8780-8785.

17. Weinberg, E.D. 1986. Iron, infection and neoplasia. Clin. Physiol. Biochem. 4:50-60.

18. Park, C.H., Valore, E.V., Waring, A.J., and Ganz, T. 2001. Hepcidin, a urinary antimicrobial peptide synthesized in the liver. J. Biol. Chem. 276:7806-7810.

\title{
Hold the antioxidants and improve plasma lipids?
}

\author{
Ronald M. Krauss
}

Children's Hospital Oakland Research Institute, Oakland, California, USA.

\begin{abstract}
Intrahepatic proteolysis is a major determinant of secretion of ApoB-containing lipoproteins into plasma. Stimulation of post-ER presecretory proteolysis (PERPP) of ApoB by n-3 polyunsaturated fatty acids has been found to result in reduced secretion of VLDL particles by hepatocytes. A new study has shown that this stimulation is promoted by pro-oxidant conditions that result in increased hepatic lipid hydroperoxide content (see the related article beginning on page 1277). Conversely, PERPP is suppressed by antioxidants and by saturated fatty acids, which are not susceptible to lipid peroxidation. Hence reduction of oxidative stress may have the unexpected side effect of increasing plasma lipid levels.
\end{abstract}

Dietary fats with differing fatty acid composition can influence plasma lipid levels by modulating hepatic production and clearance of lipoproteins (1), as well as by altering activity of cholesteryl ester transfer protein (2). Suppression and stimulation of hepatic LDL receptor activity are major determinants, respectively, of the effects of saturated and polyunsaturated fatty acids on plasma LDL clearance (1), but the mechanisms for effects of specific fatty acids on hepatic lipoprotein production are less well understood. This is in large part due to the multiple influences of fatty acids on processes that regulate hepatic lipid production and storage, and processing of $\mathrm{ApoB}$ in conjunction with lipoprotein synthesis and secretion (3).

Fatty acids are critically involved in hepatic lipoprotein production pathways that help maintain hepatic cholesterol homeostasis and the ability to respond to energy and other metabolic needs. Triglycerides influence a critical early step in secretion of ApoB-containing lipoproteins, namely the cotranslational binding of lipids to ApoB in the ER mediated by micro-

Nonstandard abbreviations used: ER-associated degradation (ERAD); microsomal triglyceride transfer protein (MTP); post-ER presecretory proteolysis (PERPP); thiobarbituric acid-reactive substance (TBARS).

Conflict of interest: The author has declared that no conflict of interest exists.

Citation for this article: J. Clin. Invest. 113:1253-1255 (2004). doi:10.1172/JCI200421637. somal triglyceride transfer protein (MTP). The resulting protection of specific ApoB domains from proteolysis, termed ER-associated degradation (ERAD), leads either to secretion of a relatively lipid-depleted particle or to further, posttranslational lipidation (3) (Figure 1). The latter may occur in a graded manner in the ER, the vesicular tubular complex, and/or the Golgi apparatus, or by fusion with a preformed lipid droplet in the ER by a process that is not dependent on MTP.

\section{Post-ER presecretory proteolysis and} hepatic lipid hydroperoxide content

Recently, Fisher et al. have identified another degradative process that can modulate hepatic secretion of more mature ApoB-containing lipoproteins (4). They have found that inhibition of hepatic ApoB secretion by $n-3$ polyunsaturated fatty acids occurs via activation of this process, which has been designated postER presecretory proteolysis (PERPP) (4). In this issue of the JCI, Pan, Fisher, and colleagues have now shown, using several lines of evidence, that this effect is mediated by fatty acid peroxidation, and that it also occurs with n-6 polyunsaturated fatty acids (5). Moreover, the finding that ApoB degradation is stimulated by pro-oxidant conditions and inhibited by antioxidants raises the question of whether variation in intrahepatic oxidative stress contributes to physiologic and/or pathologic modulation of ApoB-containing lipoprotein metabolism. There is indeed abundant evidence that consumption of supplements of longer-chain n-3 marine-derived polyunsaturated fatty acids (eicosapentaenoic and docosahexanoic acids) can lower plasma triglyceride and VLDL levels in humans (6). Recently, it has been shown that population variation in dietary intake of a plantderived n-3 polyunsaturated fatty acid, linolenic acid, is significantly associated with plasma triglyceride levels, independent of other nutrients, including longerchain n-3 polyunsaturated fatty acids (7). As Pan et al. point out, however, in their study linolenic acid suppressed ApoB secretion from rat hepatoma cells to a greater extent than could be accounted for by the relation of secretion to intrahepatic lipid hydroperoxide content as assessed by levels of thiobarbituric acid-reactive substances (TBARSs) (5). It should be noted, however, that TBARSs do not represent the full spectrum of products of lipid peroxidation, such as F2-isoprostanes. Moreover, despite the capacity for peroxidation of both n-3 and n-6 polyunsaturated fatty acids, the latter have minimal and generally nonsignificant effects on plasma triglyceride levels in humans (8). Hence increased lipid peroxidation of n-3 fatty acids does not fully explain the effects of these fatty acids on hepatic ApoB-containing lipoprotein secretion. Other effects, such as reduced lipid synthesis, may also play a role.

\section{Oxidative stress and modulation of pathways for hepatic lipoprotein secretion}

The direct relation of the number of unsaturated bonds to lipid peroxidation is consistent with the lack of a suppressive effect of saturated fatty acids on plasma triglyceride levels. In contrast, a saturated fatty acid, myristic acid, was found to stimulate secretion of ApoB-containing 


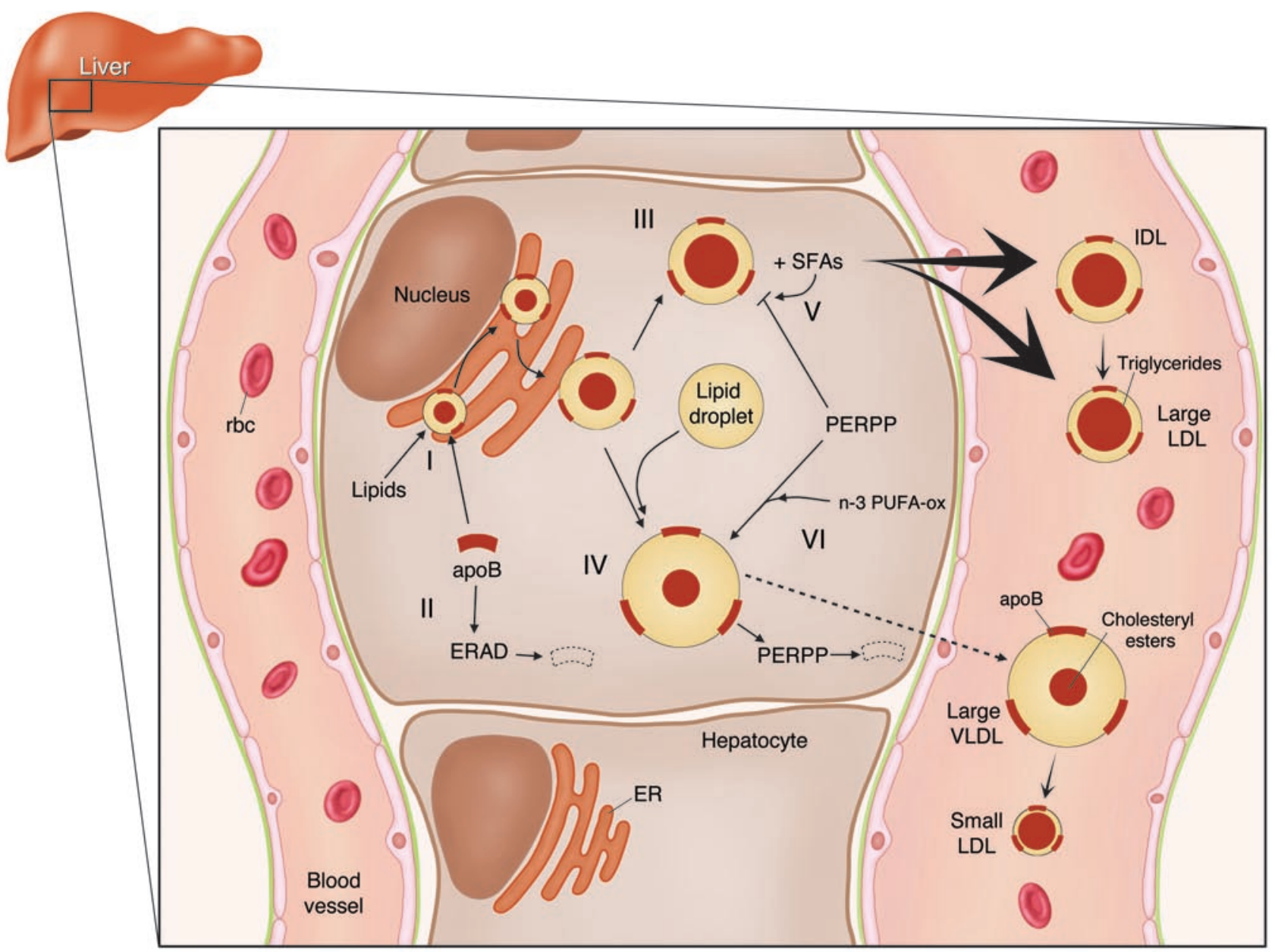

Figure 1

Scheme for the roles of intracellular proteolytic processes in regulating pathways for hepatic secretion of ApoB-containing lipoproteins and the modulating effects of saturated and n-3 fatty acids on these processes. Cotranslational lipidation of ApoB protects nascent particles from ERAD. Maturing particles can acquire choleteryl ester and be secreted as IDLs and larger LDLs or can fuse with a preformed lipid droplet to form larger VLDLs. Saturated fatty acids (SFAs) protect the smaller particles from PERPP, leading to increased secretion. Peroxidation products of $n-3$ polyunsaturated fatty acids (n-3 PUFA-ox), and perhaps also n-6 polyunsaturated fatty acids (not shown), increase PERPP, leading to decreased secretion of larger VLDLs and hence lower levels of their catabolic products, including small LDL particles.

lipoproteins from liver cells in conjunction with protection from a proteolytic process consistent with PERPP (9). Interestingly, the secreted lipoproteins under these conditions were denser than VLDLs and were more triglyceride-depleted than those secreted in the presence of albumin or oleic acid. This suggests that differences in conformation or composition of newly synthesized ApoB-containing lipoproteins can alter their exposure to PERPP in such a way that smaller, triglyceride-depleted particles formed under the influence of saturated fatty acids tend to escape this process, while larger, triglyceride-rich VLDLs are more susceptible. However, the results of Pan et al. (5) indicate that the stimulation of lipoprotein secretion by myristic acid is disproportionately greater than would be predicted by reduced hepatic TBARS content, suggesting, as was the case with linolenic acid, that effects other than those related to lipid peroxidation are involved.

Many questions remain as to the nature and physiologic importance of the PERPP system, the properties of lipoproteins that affect their susceptibility to PERPP, the mechanisms by which dietary fatty acids can affect this susceptibility, and the means by which oxidation products can suppress PERPP. It will be particularly challenging to assess the extent to which these mechanisms may influence metabolism of ApoBcontaining lipoproteins in humans. However, clues may be found in evidence that levels and distribution of LDL subspecies can serve as markers for intrahepatic pathways that result in formation of different hepatic secretory products (10) (Figure 1).

We have found that increased intake of saturated fatty acids is associated with higher levels of larger, more buoyant LDL particles (11). The concentrations of large, buoyant LDL particles are inversely related to plasma VLDL levels (12). Lipoprotein kinetic studies in humans have indicated that reduced plasma triglyceride and VLDL levels are correlated with the extent of direct secretion of IDLs and LDLs into plasma (13). Hence, one might surmise (Figure 1) that increased intake of cholesterol-raising saturated fatty acids increases direct hepatic secretion of IDLs and large LDLs, a process consistent with the evi- 
dence cited above that such secretion may be facilitated by protection from PERPP. Similarly, increased intake of $\omega-3$ fatty acids has been shown to result in a shift from small, dense LDLs to large, buoyant LDLs (14). This effect is likely to be multifactorial, with an important role for reduced cholesteryl ester transfer proteinmediated transfer of triglyceride to LDLs and subsequent lipolysis $(10,13)$. However, it is also consistent with evidence that larger, triglyceride-rich VLDLs are metabolic precursors of smaller LDL particles (10), and with the findings of Pan et al. (5) that increased PERPP reduces hepatic output of particles in this pathway (Figure 1).

A possible role for oxidation products in modulating hepatic lipoprotein secretion in humans can be assessed by determination of whether antioxidant treatment increases plasma transport or concentrations of ApoB-containing lipoproteins. In a randomized placebo-controlled study of 20,536 adults with pre-existing vascular disease or diabetes, daily supplementation with $600 \mathrm{mg}$ vitamin E, $250 \mathrm{mg}$ vitamin C, and $20 \mathrm{mg} \beta$-carotene resulted in small but statistically significant increases in triglyceride (11\%), ApoB (5\%), and LDL cholesterol (3\%), along with a small reduction in HDL cholesterol (15). No changes in triglyceride or LDL levels following antioxidant supplementation were found in a much smaller trial, but there was a reduction in the HDL2 cholesterol fraction, which was associated with reduced benefit on coronary disease endpoints when antioxidants were combined with simvastatin plus niacin therapy (16). Thus, although there is considerable evidence for the involvement of oxidative stress in many disease processes, including atherosclerosis, the potential for unintended outcomes of antioxidant therapy should serve as a warning against proceeding with such treatment in the absence of clinicaltrial evidence for benefit and safety.

Address correspondence to: Ronald M. Krauss, Children's Hospital Oakland Research Institute, 5700 Martin Luther King, Jr., Way, Oakland, California 94609, USA. Phone: (510) 450-7908; Fax: (510) 450-7909; E-mail: rmkrauss@lbl.gov.

1. Woollett, L.A., Spady, D.K., and Dietschy, J.M. 1992. Saturated and unsaturated fatty acids independently regulate low density lipoprotein receptor activity and production rate. J. Lipid Res. 33:77-88.

2. Kurushima, H., et al. 1995. Opposite effects on cholesterol metabolism and their mechanisms induced by dietary oleic acid and palmitic acid in hamsters. Biochim. Biophys. Acta. 1258:251-256.

3. Fisher, E.A., and Ginsberg, H.N. 2002. Complexity in the secretory pathway: the assembly and secretion of apolipoprotein B-containing lipoproteins. J. Biol. Chem. 277:17377-17380.

4. Fisher, E.A., et al. 2001. The triple threat to nascent apolipoprotein B. Evidence for multiple, distinct degradative pathways. J. Biol. Chem. 276:27855-27863.

5. Pan, M., et al. 2004. Lipid peroxidation and oxidant stress regulate hepatic apolipoprotein B degradation and VLDL production. J. Clin. Invest. 113:1277-1287. doi: $10.1172 / 200419197$.

6. Kris-Etherton, P.M., Harris, W.S., and Appel, L.J.
2003. Fish consumption, fish oil, omega-3 fatty acids, and cardiovascular disease. Arterioscler. Thromb. Vasc. Biol. 23:e20-e30.

7. Djousse, L., et al. 2003. Dietary linolenic acid is inversely associated with plasma triacylglycerol: the National Heart, Lung, and Blood Institute Family Heart Study. Am. J. Clin. Nutr. 78:1098-1102.

8. Mensink, R.P., Zock, P.L., Kester, A.D., and Katan, M.B. 2003. Effects of dietary fatty acids and carbohydrates on the ratio of serum total to HDL cholesterol and on serum lipids and lipoproteins: a meta-analysis of 60 controlled trials. Am. J. Clin. Nutr. 77:1146-1155.

9. Kummrow, E., Hussain, M.M., Pan, M., Marsh, J.B., and Fisher, E.A. 2002. Myristic acid increases dense lipoprotein secretion by inhibiting apoB degradation and triglyceride recruitment. J. Lipid Res. 43:2155-2163.

10. Berneis, K.K., and Krauss, R.M. 2002. Metabolic origins and clinical significance of LDL heterogeneity. J. Lipid Res. 43:1363-1379.

11. Dreon, D.M., et al. 1998. Change in dietary saturated fat intake is correlated with change in mass of large low-density-lipoprotein particles in men. Am. J. Clin. Nutr. 67:828-836.

12. Krauss, R.M., Lindgren, F.T., and Ray, R.M. 1980. Interrelationships among subgroups of serum lipoproteins in normal human subjects. Clin. Chim. Acta. 104:275-290.

13. Packard, C.J., et al. 2000. Apolipoprotein B metabolism and the distribution of VLDL and LDL subfractions. J. Lipid Res. 41:305-318.

14. Calabresi, L., Donati, D., Pazzucconi, F., Sirtori, C.R., and Franceschini, G. 2000. Omacor in familial combined hyperlipidemia: effects on lipids and low density lipoprotein subclasses. Atherosclerosis. 148:387-396.

15. Heart Protection Study Collaborative Group. 2002. MRC/BHF Heart Protection Study of antioxidant vitamin supplementation in 20,536 high-risk individuals: a randomised placebo-controlled trial. Lancet. 360:23-33.

16. Brown, B.G., et al. 2001. Simvastatin and niacin, antioxidant vitamins, or the combination for the prevention of coronary disease. N. Engl. J. Med. $345: 1583-1592$

\title{
Dysbindin-1 and schizophrenia: from genetics to neuropathology
}

\author{
Michael J. Owen, Nigel M. Williams, and Michael C. O'Donovan
}

Department of Psychological Medicine, University of Wales College of Medicine, Cardiff, United Kingdom.

\begin{abstract}
The gene encoding dysbindin-1 has recently been implicated in susceptibility to schizophrenia. In this issue of the JCI, Talbot et al. show that, contrary to expectations, dysbindin-1 is located presynaptically in glutamatergic neurons and is reduced at these locations in schizophrenia (see the related article beginning on page 1353). Further studies of dysbindin-1 and the proteins with which it interacts can be expected to throw light on the pathogenesis of schizophrenia.
\end{abstract}

Nonstandard abbreviations used: hippocampal formation (HF).

Conflict of interest: The authors have declared that no conflict of interest exists.

Citation for this article: J. Clin. Invest. 113:1255-1257 (2004). doi:10.1172/JCI200421470.
Schizophrenia is a common, severely disabling, mental disorder (1), and understanding its etiology and pathogenesis is one of the most important challenges facing psychiatry. Despite great endeavor, achieving this has proven difficult given the absence of a diagnostic neuropathology or biological markers, and few clear insights have emerged. However, there is currently a consensus that the disorder is, at least in part, neurodevelopmental (2). At the structural level, there are reductions in the neuropil and neuronal size that are widespread but not uniform, with temporal lobe structures, notably the hippocampal formation (HF), particularly affected (3). These changes in turn probably result from alterations in synaptic, dendritic, and axonal organization (3). At the functional level, accumulating evidence also implicates altered glutamate 